\title{
Searching for Majorana neutrinos with double beta decay and with beta beams
}

\author{
L. Lukaszuk, Z. Sujkowski ${ }^{\mathrm{a}}$, and S. Wycech \\ The Andrzej Soltan Institute for Nuclear Studies, 05-400 Otwock - Świerk, Poland
}

Received: 11 July 2005 /

Published online: 14 March 2006 - (c) Società Italiana di Fisica / Springer-Verlag 2006

\begin{abstract}
The leading questions in today neutrino physics concern the transformation properties of neutrinos under charge conjugation, the lepton number conservation hypothesis and the values of neutrino masses. The answers can be obtained if the neutrinoless double beta decay, $0 \nu 2 \beta$, is observed and its rate is measured. The mere observation of the process proves the Majorana nature of neutrinos as well as the violation of the lepton number conservation, while the rate is a sensitive measure of the neutrino mass. The search for this effect at present concerns mainly the double $\beta^{-}$decay. This paper describes the recent proposal to search for the neutrinoless radiative double electron capture as an attractive alternative to the double $\beta^{-}$decay. It is shown, moreover, that the same information which is expected from the $0 \nu 2 \beta$ studies can in principle be also obtained from experiments using intense $\nu_{e}$ beams produced by radioactive ions decaying in flight (the "beta beams").
\end{abstract}

PACS. $13.15 .+\mathrm{g}$ Neutrino interactions - 23.40.Bw Weak-interaction and lepton (including neutrino) aspects $-14.60 . \mathrm{Pq}$ Neutrino mass and mixing

\section{Introduction}

The discovery of lepton flavour mixing for neutrinos, the so-called neutrino oscillations, has put the neutrino physics in the lime-light of interest of particle, astro-particle and nuclear physics. Although the number of answered questions is impressive, so is the shopping list of essential queries. Thus we know that there are three and only three kinds of weakly interacting neutrinos, we know that neutrinos are massive, we know the splittings between square masses. This paper considers some possibilities of getting answers to three of the remaining questions: the absolute mass values, the lepton number conservation and the neutrino-antineutrino identity.

The present limits on the mass values stem mainly from three sources: the cosmologic one, giving the sum of the three neutrino masses in a strongly model-dependent way (see, e.g., [1]), the direct measurement [2] of the end point of the ${ }^{3} \mathrm{H} \beta^{-}$spectrum $\left(m_{\nu_{e}} \leq 2.2 \mathrm{eV}\right)$ and the neutrinoless double $\beta$ decay $0 \nu 2 \beta\left(m_{\nu_{e}}<0.3-1.0 \mathrm{eV}\right.$, where the range reflects the large nuclear matrix element uncertainty [3,4]; a claim for having observed the effect corresponding to $(0.2-0.6) \mathrm{eV}$ neutrino mass value is made in [5]; see [3] for comments). It should be emphasized that the mere observation of this decay will provide unambiguous answers to the next two questions: it will prove the

\footnotetext{
${ }^{a}$ e-mail: sujkowsk@ipj.gov.pl
}

Majorana nature of neutrinos as well as the violation of the lepton number conservation law.

After briefly reviewing the $0 \nu 2 \beta$ method in general we shall concentrate on the recent suggestion of studying the radiative neutrinoless double electron capture as an attractive alternative to the double $\beta^{-}$emission $[6,7]$. We shall also outline the possible experiments with beta-beam based neutrino factories. We shall show that the information obtainable from such experiments is at least in principle equivalent to that from the double beta decay.

\section{The neutrinoless double beta processes}

The double beta decay processes are very slow. They are observable only in cases for which the single $\beta^{ \pm}$or EC decays are energetically forbidden. We may distinguish the following decays:

$-\beta^{-} \beta^{-}:(Z, N) \rightarrow(Z+2, N-2)+\beta^{-}+\beta^{-}+\overline{\nu_{e}}+\overline{\nu_{e}}$,

$-\beta^{+} \beta^{+}:(Z, N) \rightarrow(Z-2, N+2)+\beta^{+}+\beta^{+}+\nu_{e}+\nu_{e}$,

$-\beta^{+} \mathrm{EC}:(Z, N) \rightarrow(Z-2, N+2)+\beta^{+}+\nu_{e}+\nu_{e}$,

- ECEC $:(Z, N) \rightarrow(Z-2, N+2)+\nu_{e}+\nu_{e}$.

If neutrino is a massive Majorana particle then the first three processes can proceed without neutrino emission: the neutrino emitted in one weak interaction vertex is absorbed in the other one and only the $\beta^{-}$or $\beta^{+}$electrons are emitted. These carry out the total energy excess. The double electron capture decay is not possible without 


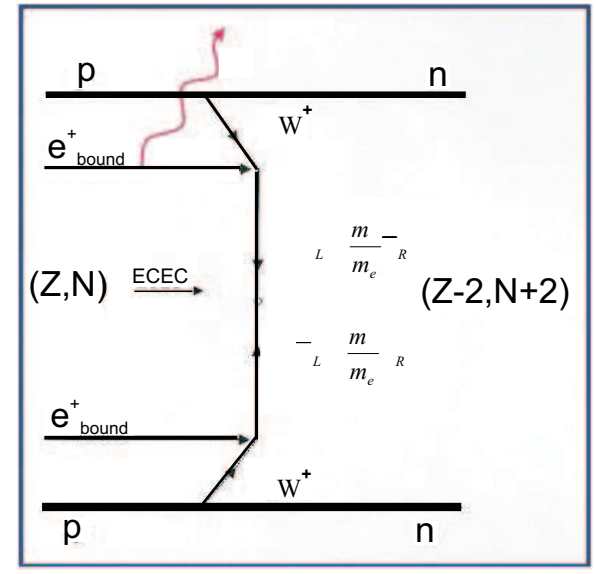

Fig. 1. Diagram for radiative neutrinoless double electron capture.

an extra energy carrier. The most obvious one is a single photon radiated by one of the captured electrons. This is the radiative neutrinoless double electron capture, see fig. 1, discussed in some details below.

The value to be determined in any of the $0 \nu 2 \beta$ processes is the rate. This is a sensitive measure of the neutrino mass. To a good approximation the rate can be factorized:

$$
\Gamma=G(E, Z) M^{2} X^{2},
$$

where $G(E, Z)$ is the phase space factor which can be calculated rather accurately, $M$ is the nuclear matrix element, not so easy to calculate, and $X$ is proportional to the effective neutrino rest mass.

For a detailed discussion of the $0 \nu 2 \beta$ processes, and especially of the best studied $0 \nu \beta^{-} \beta^{-}$one, we refer the reader to recent reviews $[3,4]$. Below, we return to the radiative neutrinoless double electron capture, $0 \nu \mathrm{ECEC} \gamma$. The process is possible due to the admixture of neutrinos of opposite helicity. The amplitudes of these admixtures are proportional to the neutrino rest mass. It should be noted that because of the angular momentum conservation requirement the process precludes the capture of two $1 S$ electrons. It is either the $1 S+2 S$ electrons (the magnetic type transition) or the $1 S+2 P$ electrons (the electric dipole transition). It is the second case which is of particular interest because of the possible atomic resonance enhancement of the rate.

The theory of radiative single electron capture has been given by Glauber and Martin in the fifties [8]. The continuous photon spectra of this internal bremsstrahlung process exhibit an interesting structure in the case of the $2 P$ (or $3 P$ ) electron capture: there is a singularity at the photon energy equal to the $1 S-2 P$ or $1 S-3 P$ atomic level energy difference. This prediction has been confirmed experimentally for several cases (cf., e.g., [9]). The same approach can be applied to the $0 \nu$ ECEC $\gamma$ case. Figure 2 shows the Feynman diagram for such a resonance situation. The process can be visualised as follows: after the second $1 S$ electron is virtually captured, the system lives long enough for one of the $1 S$ holes to be filled by the $2 P$

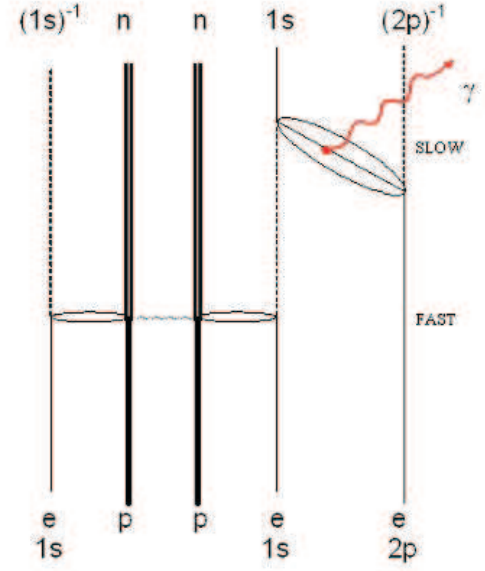

Fig. 2. Diagram for atomic resonance in radiative double electron capture.

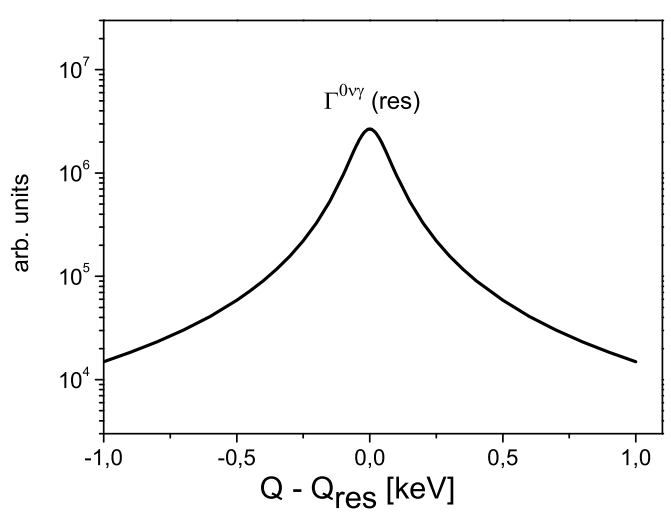

Fig. 3. The rate $v s$. energy dependence of $0 \nu \operatorname{ECEC} \gamma$ process in vicinity of the atomic resonance for a high $Z$ atom.

electron. The resonance condition is fulfilled if the energy of the resulting $\mathrm{K} \alpha \mathrm{X}$-ray transition coincides with the energy available for the internal bremsstrahlung photon. The rate can be expressed [6] as

$$
\Gamma^{0 \nu \gamma}(Q)=\frac{\Gamma^{r}(2 P \rightarrow 2 S)}{\left[Q-Q_{r e s}(Z-2)^{2}\right]+\left[\frac{\Gamma^{r}}{2}\right]^{2}}\left|R_{0 \nu}\right|^{2},
$$

where $Q$ is the available bremsstrahlung energy $Q_{r e s}(Z-$ 2 ) is the $\mathrm{K} \alpha \mathrm{X}$-ray energy in the daughter atom and $\Gamma_{r}$ is the natural width of the X-ray transition in the presence of an additional $1 S$ hole (the width of a hypersatellite transition, of the order of $100 \mathrm{eV}$ in heavy atoms).

Figure 3 shows the rate of the $0 \nu \mathrm{ECEC} \gamma$ process in vicinity of the resonance. The enhancement attainable in heavy emitters may reach in favourable cases several orders of magnitude. This more than compensates the usual retardate of a radiative process. For details of the theory of the $0 \nu$ radiation double electron capture we refer to $[6,7,10,11]$. Here we can mention that the process has several experimental advantages, the most important of which being the precious coincidence trigger. The trigger 
is due to the presence of characteristic $\mathrm{K} \alpha \mathrm{X}$-rays and, in cases of decays to excited states, of characteristic $\gamma$-rays de-exciting these states. The resonance conditions favour in many cases decays to such states, while in the $\beta^{-} \beta^{-}$ case the $Q^{5}$-dependence of the rate strongly inhibits the process. Without a coincidence trigger the requirements of shielding and purity of all the material, necessary in order to suppress the overwhelming random background, tend to the extremes. Other advantages such as, e.g., the low physical background due to the competing two neutrino radiative process, are discussed in $[6,10]$.

There are several candidates for $0 \nu$ ECEC decays which might meet the resonance condition. The rate estimates, however, suffer from large uncertainties due to low precision of mass measurements, typically a few $\mathrm{keV}$ to be compared with the resonance width of $\sim 0.1 \mathrm{keV}$.

Mass measurements of the required accuracy are within the present state-of-the art, but have yet to be done. The nuclei to be considered are, e.g. ${ }^{112} \mathrm{Sn},{ }^{136} \mathrm{Ce}$, ${ }^{152} \mathrm{Gd},{ }^{162} \mathrm{Er},{ }^{164} \mathrm{Er}$ and ${ }^{180} \mathrm{~W}$. The corresponding $Q-Q_{\text {res }}$ energy values in $\mathrm{keV}$ are $-5.8(4.6), 27(13), 0.04(3.5)$, $-10.5(4), 5(4), 12(5)$, where $Q$ is the decay energy available for the process [12] and $Q_{r e s}$ is the resonance energy; see [6] for details.

\section{Searching for Majorana neutrinos with beta beams}

\subsection{Beta beams}

The present plans for developing high flux, high energy neutrino beams, the so-called "neutrino factories", are mainly motivated by the need for the next generation, high precision neutrino oscillation experiments. Once constructed, however, these facilities will inevitably be used for a number of other research projects. The principle is to produce a high intensity beam of a neutrino progenitor, be it muons or beta-decaying ions, accelerate these species to highly relativistic energies and store in a ring ([13,14, 15] and references therein). The "ring" will have a long straight-linear section. The neutrinos produced during the flight in this section will undergo a kinematical focusing and a relativistic energy boost. The former concentrates the neutrino beam at the detector (the "target"), the latter offers a dramatic increase of the interaction crosssection in the detector. The purpose of the present paper is to consider the use of the beta beam born neutrinos to study other fundamental properties of neutrinos: their Majorana or Dirac nature, the lepton number conservation hypothesis and the absolute mass scale.

\subsection{The principle of experiment}

The Majorana neutrinos produced in $\beta^{-}$or in $\beta^{+}$, EC decays differ only by their helicity. If $\nu$ is massive then in either kind of the decay there is an admixture of the helicity opposite to the dominating one. The magnitude of this admixture depends on the neutrino rest mass, $m_{\nu}$, and its energy in the center of mass frame of the decaying species, $E_{\nu}^{\mathrm{CM}}$ :

$$
f\left(m_{\nu}, E_{\nu}^{\mathrm{CM}}\right) \sim\left(\frac{m_{\nu}}{2 E_{\nu}^{\mathrm{CM}}}\right)^{2} .
$$

We assume having

i) beams of either $\nu_{e}$ or $\overline{\nu_{e}}$ with $100 \%$ purity

ii) detectors with $100 \%$ selectivity to neutrinos of a given helicity.

We consider two situations:

a) single beam, two different detectors

b) single detector, two different beams.

For the purpose of the present argument these situations are equivalent.

Consider the case of the single $\nu$ beam and two different detectors labelled $(\operatorname{det} \nu)$ and $(\operatorname{det} \bar{\nu})$. (In the following we use the notation $\nu, \bar{\nu}$ for the neutrinos with dominating left or right handed helicity, respectively.) The corresponding rates observed by the two detectors are:

$$
\begin{aligned}
& N_{\nu}(\operatorname{det} \nu) \sim N_{\nu}^{0} \sigma_{\nu n}\left(E_{\nu}^{l a b}\right), \\
& N_{\nu}(\operatorname{det} \bar{\nu}) \sim N_{\nu}^{0} \sigma_{\bar{\nu} p}\left(E_{\nu}^{l a b}\right) f\left(m_{\nu}, E_{\nu}^{\mathrm{CM}}\right),
\end{aligned}
$$

where $N_{\nu}^{0}$ is the neutrino flux at the detector, $E_{\nu}^{l a b}$ is the boosted neutrino energy in the laboratory frame and $\sigma_{\nu n}\left(E_{\nu}^{l a b}\right), \sigma_{\bar{\nu} p}\left(E_{\nu}^{l a b}\right)$ are the cross-sections for the boosted neutrinos of the dominating and the admixed helicity, respectively. The admixed fraction, $f$, can then be expressed by the measured and/or calculated quantities:

$$
f\left(m_{\nu}, E_{\nu}^{\mathrm{CM}}\right)=\frac{N_{\nu}(\operatorname{det} \bar{\nu})}{N_{\nu}(\operatorname{det} \nu)} \frac{\sigma_{\bar{\nu} p}\left(E_{\nu}^{l a b}\right)}{\sigma_{\nu n}\left(E_{\nu}^{l a b}\right)} \frac{\varepsilon_{\nu n}\left(E_{\nu}^{l a b}\right)}{\varepsilon_{\bar{\nu} p}\left(E_{\nu}^{l a b}\right)},
$$

where $\varepsilon_{\nu n}, \varepsilon_{\bar{\nu} p}$ denote the detector efficiencies. The notation corresponds to the vector current interactions $(\nu-\mathrm{n})$ and $(\bar{\nu}-\mathrm{p})$, respectively. Note that for any observation angle there is a continuous energy spectrum depending on energy and emission angle in the CMS.

The detection cross-sections can be calculated, albeit with sizeable nuclear structure uncertainties. Alternatively, they can be determined experimentally. In the ideal case of the experiment done with $\bar{\nu}$ and $\nu$ beams of the same energy and with the two different detectors, the $f\left(m_{\nu}, E_{\nu}^{\mathrm{CM}}\right)$ function can be expressed in a simplified way. The terms of the detector efficiencies and the crosssections (and thus of the nuclear structure dependence) cancel:

$$
f\left(m_{\nu}, E_{\nu}^{\mathrm{CM}}\right)=\frac{N_{\nu}(\operatorname{det} \bar{\nu})}{N_{\nu}(\operatorname{det} \nu)} \frac{N_{\bar{\nu}}(\operatorname{det} \nu)}{N_{\bar{\nu}}(\operatorname{det} \bar{\nu})} .
$$

\section{Helicity admixture estimates}

\subsection{Admixture in the $\mathrm{CM}$ system}

Consider the ${ }^{6} \mathrm{He}$ decay (this is the prime candidate presently proposed for the beta beam, see the CERN feasibility study, [16]):

$$
{ }^{6} \mathrm{He} \rightarrow{ }^{6} \mathrm{Li}+\bar{\nu}+\mathrm{e}^{-} .
$$




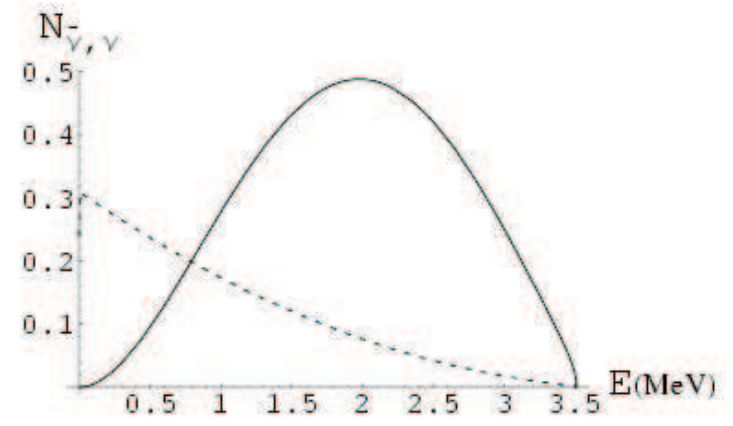

Fig. 4. The $\bar{\nu}$ energy spectrum (solid) and the opposite helicity admixture (dashed, $\times 10^{13}$ ) for ${ }^{6} \mathrm{He}$ decay in CM system. $N_{\nu}$ in arbitrary units.

The maximum $\bar{\nu}$ energy is about $3.5 \mathrm{MeV}$. The spectrum is shown in fig. 4 . The spectrum of neutrinos with opposite helicity, calculated for $m_{\bar{\nu}}=0.5 \mathrm{eV}$ and multiplied with $10^{13}$ is shown with the dashed line.

The neutrino CM energy dependence indicates the need to look for ions with decay energies as low as possible. A possible candidate is ${ }^{178} \mathrm{~W}$ decaying via the electron capture decay:

$$
\begin{aligned}
& { }_{74}^{178} \mathrm{~W}^{\mathrm{EC}} \rightarrow{ }_{74}^{178} \mathrm{Ta} \\
& \text { with } Q(\mathrm{EC})=91.3 \mathrm{keV} .
\end{aligned}
$$

The neutrino emitted in the CM system is mono-energetic, with the energy

$$
E_{\nu}^{\mathrm{CM}}=Q-B_{K}=23.9 \mathrm{keV}
$$

for neutral ${ }^{178} \mathrm{~W}$ and $\simeq 22 \mathrm{keV}$ for the ${ }_{74}^{178} \mathrm{~W}^{73+}$ ion. The gain has to be weighted, however, against the loss in intensity due to the elongated lifetime of the relativistic emitter.

\subsection{Helicity admixture in the boosted system. The "helicity flip"}

The helicity composition of the boosted beam of massive neutrinos will differ from that in the CM system. This comes about from the "helicity flip" of the neutrinos emitted in $\mathrm{CM}$ and reoriented due the boost in laboratory. Similar effect has been considered in [17] for pion decay. The very tiny rest mass of neutrinos, however, means that the gain in the helicity admixture is not very spectacular, at least not for the boosts presently attainable.

The ratio $R$ of boosted admixture to that in the CMS can be very well approximated for a large range of laboratory energies by the formula [18]:

$$
R=\frac{E_{\max }}{E^{l a b}}=2 \gamma E^{\mathrm{CM}} / E^{l a b}
$$

Taking a mono-energetic neutrino, e.g., $25 \mathrm{keV}$ in CMS, and $\gamma=1000$ one gets $E_{\max }=50 \mathrm{MeV}$ and thus at $E^{l a b}=1 \mathrm{MeV}$ the gain factor $R=50$.

\section{Conclusions and outlook}

- Neutrino oscillations prove that at least one of the neutrino species has a finite rest mass and that the lepton flavour is not conserved. Information from other kind of data is needed to determine the values of neutrino masses, to establish the mass hierarchy and to test total lepton number conservation hypothesis.

- Neutrinoless double beta decay offers the most sensitive way to determine the effective neutrino mass. If discovered, it proves unambiguously the Majorana nature of neutrinos as well as the lepton number nonconservation.

- The radiative neutrinoless double electron capture is a promising alternative to the electron emission, with definite experimental advantages.

- Strong, resonance enhancement is predicted at photon energy equal to the energy difference of the $1 S-2 P$ atomic state.

- Experiments with beta beams can in principle provide information equivalent to that from the double beta decay. The rates are discouraging but improvements possible.

\section{References}

1. H. Ejiri, I. Ogawa (Editors), Proceedings of the 1st $\mathrm{Ya}$ mada Symposium on Neutrinos and Dark Matter in Nuclear Physics, Nara, Japan, 2003, unpublished.

2. V. Lobashev et al., Nucl. Phys. (Proc. Suppl.) 91, 280 (2001); C. Weinheimer et al., International Conference on Neutrino Physics and Astrophysics Neutrino'02, May 2002, Munich; N. Bohr, Mat.-Fys. Medd. K. Dan. Vidensk. Selsk. 18, No. 8 (1948).

3. S. Elliott, P. Vogel, Annu. Rev. Nucl. Part. Sci. 52, 15 (2002); S.R. Elliott, J. Engel, J. Phys. G 30, R183 (2004).

4. Z. Sujkowski, Acta Phys. Pol. B 34, 2207 (2003).

5. H.V. Klapdor-Kleingrothaus et al., Part. Nucl. Lett. 1, 20 (2001); Phys. Lett. B 586, 198 (2004); H.V. KlapdorKleingrothaus, Nucl. Phys. B 143, 229 (2005).

6. Z. Sujkowski, S. Wycech, Phys. Rev. C 70, 052501(R) (2004).

7. Z. Sujkowski, S. Wycech, Nucl. Instrum. Methods B 235, 81 (2005).

8. R.J. Glauber, P.C. Martin, Phys. Rev. 104, 158 (1956); P.C. Martin, R.J. Glauber, Phys. Rev. 109, 1307 (1958).

9. J. Żylicz et al., Nucl. Phys. 42, 330 (1963).

10. S. Wycech, Z. Sujkowski, Acta Phys. Pol. B 35, 1223 (2004).

11. J. Bernabeu, A. de Rujula, C. Jarlskog, Nucl. Phys. B 223 , 15 (1983).

12. G. Audi, A.H. Wapstra, C. Thibault, Nucl. Phys. A 729, 337 (2003).

13. F.T. Avignone et al., Phys. At. Nucl. 63, 1007 (2000); http://www .phy.ornl.gov.orland; see also http://www . pas.rochester.edu/ksmcf/minerva.

14. P. Zucchelli, Phys. Lett. B 532, 166 (2002).

15. J. Serreau, C. Volpe, Phys. Rev. C 70, 055502 (2004).

16. B. Autin et al., J. Phys. G 29, 1785 (2003); see also CERN Workshop Physics with multi-MW proton source, CERN, May 2004.

17. P. Langacker, J. Wong, Phys. Rev. D 58, 093004 (1998).

18. L. Łukaszuk, Z. Sujkowski, S. Wycech, to be published. 